\title{
TFEC contributes to cardiac hypertrophy by inhibiting AMPK/mTOR signaling
}

\author{
TING ZHAO* , ZHENYU WANG ${ }^{*}$, YEHONG CHI, CHUNMEI NI and XUDAN ZHENG \\ Department of Cardiology, Weapon Industry 521 Hospital, Xi'an, Shanxi 710065, P.R. China
}

Received April 8, 2021; Accepted July 9, 2021

DOI: $10.3892 / \mathrm{etm} .2021 .10706$

\begin{abstract}
The underlying mechanism of cardiac hypertrophy has not yet been fully elucidated. The present study aimed to explore the function of transcription factor EC (TFEC) in mouse models of cardiac hypertrophy and to determine the underlying mechanism. Pressure-overload cardiac hypertrophy and angiotensin II (AngII) infusion-induced animal models of cardiac hypertrophy were established in vivo. The expression of TFEC was explored via western blotting. The results demonstrated that TFEC expression was significantly increased in the hearts of mice with pressure overload- and AngII-induced hypertrophy. Injection of rAd-short hairpin (sh)-TFEC significantly decreased the expression of TFEC in heart tissues compared with group injected with rAd-negative control (NC). Furthermore, the expression levels of atrial natriuretic peptide (ANP), brain natriuretic peptide $(\mathrm{BNP})$ and $\beta$-myosin heavy chain $(\beta$-MHC) were increased in the hearts of AngII-treated mice; however, compared with rAd-NC transfection, transfection with rAd-sh-TFEC decreased the expression levels of ANP, BNP and $\beta$-MHC. The results from echocardiographic analysis indicated that transfection with rAd-sh-TFEC improved the cardiac function of AngII-treated mice compared with transfection with rAd-NC. In addition, the AngII-induced increase in cardiomyocyte size could be reversed by TFEC knockdown in primary cardiomyocytes. The elevated expression levels of ANP, BNP and $\beta$-MHC induced by AngII could be partially abolished following TFEC knockdown. The results from western blotting demonstrated that TFEC overexpression decreased the expression of phosphorylated AMP-activated protein kinase (AMPK)/acetyl-CoA carboxylase (ACC) but
\end{abstract}

Correspondence to: Dr Xudan Zheng, Department of Cardiology, Weapon Industry 521 Hospital, 12 Zhangba East Road, Xi'an, Shanxi 710065, P.R. China

E-mail: ert201012@163.com

*Contributed equally

Key words: cardiac hypertrophy, kinase non-catalytic C-lobe domain containing 1, AMP-activated protein kinase/mechanistic target of rapamycin signaling increased the expression of phosphorylated mechanistic target of rapamycin (mTOR). Furthermore, Compound C significantly suppressed the activation of AMPK/ACC but increased the activation of mTOR, even in primary cardiomyocytes transfected with rAd-sh-TFEC. In conclusion, the findings from this study demonstrated that TFEC was overexpressed in the hearts of mice with cardiac hypertrophy and that silencing TFEC may improve AngII-induced cardiac hypertrophy and dysfunction by activating AMPK/mTOR signaling.

\section{Introduction}

Cardiac hypertrophy is an adaptive response to hemodynamic stress and is associated with impaired cardiac function, including an increase in cardiomyocyte size, higher sarcomere organization and enhanced protein synthesis for natriuretic peptide $\mathrm{A}(\mathrm{ANP})$, natriuretic peptide $\mathrm{B}(\mathrm{BNP})$ and $\beta$-myosin heavy chain $(\beta-\mathrm{MHC})$, and higher sarcomere organization $(1,2)$. Pathological cardiac hypertrophy is an independent risk factor for myocardial infarction, arrhythmia and heart failure (HF) (3). Without effective intervention, prolonged HF can lead to sudden death (3). The underlying mechanisms remain unclear. Therefore, in-depth studies are urgently required.

Transcription factor EC (TFEC) is a basic helix-loop-helix transcription factor that is a member of the MITF family $(4,5)$. TFEC is highly enriched in caudal endothelial cells when hematopoietic stem cells (HSCs) colonize the caudal hematopoietic tissue (6). A previous study demonstrated that TFEC facilitates HSC-derived hematopoiesis in a non-cell-autonomous fashion (6). TFEC is also expressed in bone marrow-derived macrophages following stimulation with the Th2 cytokines interleukin (IL)-4 and IL-13 or lipopolysaccharide (7). However, TFEC is suggested to be not ubiquitously expressed (8). For example, TFEC RNA is not found in several cell types, including fibroblasts, myoblasts, chondrosarcoma cells and myeloma cells (8). Whether TFEC is expressed during cardiac hypertrophy and involved in the progression of cardiac hypertrophy has never been explored.

The present study aimed to determine whether TFEC is dysregulated in mouse models of cardiac hypertrophy. In addition, this study aimed to explore the mechanism by which TFEC might be involved in the development of cardiac hypertrophy. 


\section{Materials and methods}

Animal models. Eight-week-old male adult C57BL/6 mice weighing 20-25 g $(\mathrm{n}=45)$ were purchased from Charles River Laboratories. The mice were housed under a 12-h light/dark cycle and pathogen-free conditions and were given free access to standard mouse chow and tap water. This study was approved by the research ethics committee of Weapon Industry 521 Hospital (approval no. WI-J2018923A).

Pressure-overload cardiac hypertrophy. The pressureoverload model of cardiac hypertrophy was established by transverse aortic constriction (TAC) (9). Adult mice (C57BL/6, 8 weeks old), weighing 20-25 g, were anaesthetized with pentobarbital sodium $(50 \mathrm{mg} / \mathrm{kg}$; intraperitoneal injection; $\mathrm{n}=12$ ) (10). To confirm anesthesia, the absence of withdrawal reflex in response to a tail pinch was observed. The mice were placed in the supine position. Following successful endotracheal intubation, the cannula was connected to a volume-cycled rodent ventilator. To identify the thoracic aorta, the chest was opened. Then, a 5-0 silk suture was placed below the transverse aorta and tied around a 26-gauge blunt needle, which was subsequently removed. The sham group underwent the aforementioned procedure, but did not receive a 5-0 silk suture. The chest was closed and the animals were maintained under ventilation until autonomic breathing successfully recovered. After 4 weeks, surviving animals (all but one mouse, which died during TAC surgery, survived) were analyzed by echocardiography and killed by cervical dislocation. The hearts were quickly harvested and incubated in $4 \%$ paraformaldehyde at room temperature for $20 \mathrm{~min}$ or dissected into the left ventricle (LV), right ventricle and ventricular septum, which were rapidly frozen in liquid nitrogen and stored at $-80^{\circ} \mathrm{C}$ for subsequent analyses.

The mice that survived $24 \mathrm{~h}$ after operation $(\mathrm{n}=10$ mice per group) were observed for 4 weeks. The survival rate of $10 \mathrm{C} 57 \mathrm{BL} / 6$ mice in sham group was $100 \%$. Only one of the $10 \mathrm{C} 57 \mathrm{BL} / 6$ animals undergoing TAC operation died (survival rate for TAC, 90\%). The heart and body weight (HW/BW) ratio was compared between the TAC group and sham group.

Angiotensin II (AngII) infusion-induced animal model of cardiac hypertrophy. A mouse model (C57BL/6, 8 weeks old, weighing 20-25 g) of AngII (1.46 mg/kg/day for 28 days) infusion-induced cardiac hypertrophy was established (11). Control group mice (C57BL/6; 8 weeks old; weight 20-25 g) were injected daily via intraperitoneal administration with PBS (100 $\mu$ l for 28 days). Mice were anaesthetized via intraperitoneal administration of sodium pentobarbital (50 mg/kg) (10). To confirm the anesthesia, the absence of a reflex in response to a foot squeeze was observed. Then, an AngII mini-osmotic pump (Alzet model 2002; Cupertino) was used. The body temperature was maintained at $37 \pm 0.5^{\circ} \mathrm{C}$ during the surgery. All mice were euthanized by resection of the heart and decapitation under deep isoflurane anesthesia (5\%) (12). Animal hearts were quickly harvested and incubated in $4 \%$ paraformaldehyde at room temperature for $20 \mathrm{~min}$ or dissected into the LV, right ventricle and ventricular septum, which were rapidly frozen in liquid nitrogen and stored at $-80^{\circ} \mathrm{C}$ for subsequent analyses. The ratio of $\mathrm{HW} / \mathrm{BW}$ was compared between the AngII treatment group and controls.

For the control group, all mice $(n=10)$ survived during the experiment, equating to a $100 \%$ survival rate in the $10 \mathrm{C} 57 \mathrm{BL} / 6$ mice of the control group. Only one of the 10 C57BL/6 animals undergoing AngII treatment died (survival rate for AngII treated mice, $90 \%$ ).

Cell culture. One-day-old $(\mathrm{n}=4$ in the same litter for each experiment) mice were born from the wide type 8-10 week-old female adult C57BL/6 mice (Charles River Laboratories). Pups were decapitated using sterile scissors (straight) without anaesthesia and the chest was open along the sternum to allow access to the chest cavity and the heart as previously described (13). Briefly, murine hearts were harvested, placed in ice-cold Hanks' medium and cut into pieces. The tissues were digested with trypsin and type II collagenase at $37^{\circ} \mathrm{C}$ for $15 \mathrm{~min}$. The cells were passed through a cell strainer $(200$ mesh), centrifuged at $1,000 \mathrm{x}$ g for $10 \mathrm{~min}$ at room temperature, seeded in Petri dishes and incubated for $2 \mathrm{~h}$ at $37^{\circ} \mathrm{C}$. The supernatants containing cardiomyocytes were collected and mixed in DMEM (HyClone; Cytiva). Before treatment with AngII $(1 \mu \mathrm{mol} / \mathrm{l})$ for $24 \mathrm{~h}$, the cells were cultured in DMEM containing $1 \% \mathrm{FBS}$ for $12 \mathrm{~h}$ at $37^{\circ} \mathrm{C}$.

Measurement of cell surface area. Primary cardiomyocytes were isolated as described above and previously (13) were fixed with $4 \%$ paraformaldehyde for $20 \mathrm{~min}$ at room temperature, permeabilized with $0.4 \%$ Triton X-100 (Beijing Solarbio Science \& Technology Co., Ltd.) for $1 \mathrm{~h}$ at room temperature and then blocked with 5\% goat serum (Beijing Solarbio Science \& Technology Co., Ltd.) at $37^{\circ} \mathrm{C}$ for $1 \mathrm{~h}$. Then, cells were incubated with anti-sarcomeric alpha actin antibody (1:250; cat. no. ab137346; Abcam) at $4^{\circ} \mathrm{C}$ overnight and subsequently with a DyLight 594-conjugated goat antimouse antibody (cat. no. A23610; AmyJet Scientific, Inc.) at room temperature for $1 \mathrm{~h}$. Cells were subsequently observed under a fluorescence microscope (magnification, x20).

Echocardiography study. Mice were anesthetized with isoflurane inhalation at the concentration of $2.5 \%$ for anesthetic induction and at $1 \%$ for anesthetic maintenance (14-16). Echocardiography was performed to evaluate the function of the LV using a MyLab ${ }^{\text {TM }}$ Seven VET system (Esaote SpA) equipped with a $10-\mathrm{MHz}$ probe. Parasternal short axis images at the mid-papillary muscle level were recorded in M-mode. The LV dimensions, including LV end-systolic diameter (LVEDs), LV end-diastolic diameter (LVEDd), posterior wall thickness, left ventricular ejection fractions (LVEF), maximum change in left ventricular pressure over time $(\mathrm{dp} / \mathrm{dtmax}, \mathrm{mmHg} / \mathrm{s})$ and minimum change in left ventricular pressure over time (dp/dtmin, $\mathrm{mmHg} / \mathrm{s}$ ) were measured.

Histopathology staining. The mouse hearts were fixed in $4 \%$ formalin for $20 \mathrm{~min}$ at room temperature. The heart sections were embedded in paraffin and sectioned to a thickness of $5 \mu \mathrm{m}$ for staining with hematoxylin and eosin (H\&E). Slides were stained with hematoxylin (Beijing Solarbio Science \& Technology Co., Ltd.) for 5 min and washed with tap water for $2 \mathrm{~min}$ at room temperature. The sides were differentiated with 
$1 \%$ alcohol for $2 \mathrm{~min}$ and washed with tap water for $5 \mathrm{~min}$. Subsequently, the slides were stained with eosin at room temperature for 2 min (Beijing Solarbio Science \& Technology Co., Ltd.). The slides were washed with tap water for 2 min and $70 \%$ alcohol twice, after which the slides were sealed and observed under a fluorescence microscope (magnification, x 20; BX43; Olympus Corporation). For wheat germ agglutinin (WGA) staining of cardiac muscle cell membrane, the tissue sections were stained with $1.0 \mathrm{mg} / \mathrm{ml}$ Alexa FluorVR 488-conjugated WGA (Molecular Probes) at room temperature for $20 \mathrm{~min}$ to visualize the size of cardiomyocytes using a fluorescence microscope (magnification, x20; IX71; Olympus Corporation).

Construction of adenovirus vectors. Adenovirus vectors carrying RNA for overexpression of TFEC (rAd-TFEC) or a short hairpin (sh) RNA targeting TFEC (rAd-sh-TFEC) or a negative control (rAd-NC) were constructed by Shanghai GeneChem Co., Ltd. C57BL/6 mice were injected once with vectors at a concentration of $1 \times 10^{9} \mathrm{U} / \mathrm{ml}(50 \mu \mathrm{l})$ through the left ventricular chamber after 28 days of treatment with AngII.

Reverse transcription quantitative $(R T-q) P C R$. Total RNA was extracted from the heart tissues or primary cardiomyocytes using TRIzol reagent (Invitrogen; Thermo Fisher Scientific, Inc.) and reverse transcribed into cDNA using a One Step PrimeScript ${ }^{\mathrm{TM}}$ RT-PCR Kit (Takara Bio, Inc.) according to the manufacturers' instructions. RT-qPCR analysis was performed using LightCycler 480 SYBR Green 1 Master Mix (No. 04707516001; Roche Diagnostics) according to the manufacturers' protocol. The relative mRNA expression was normalized to that of GAPDH using the $2^{-\Delta \Delta C q}$ method (17). The sequences of the primers (Sangon Biotech Co., Ltd.) were as follows: GAPDH forward, 5'-TCATCAACGGGAAGC CCATC-3', reverse, 5'-CTCGTGGTTCACACCCATCA-3'; ANP forward, 5'-ACCTGCTAGACCACCTGGAG-3', reverse, 5'-CCTTGGCTGTTATCTTCGGTACCGG-3'; BNP forward, 5'-GAGGTCACTCCTATCCTCTGG-3', reverse, 5'-GCCATT TCCTCCGACTTTTCTC-3'; $\beta$-MHC forward, 5'-CCGAGT CCCAGGTCAACAA-3', reverse, 5'-CTTCACGGGCACCCT TGGA-3'; AMP-activated protein kinase (AMPK) forward 5'-TCAAGCCCAGGACAGGATTT-3', reverse, 5'-CTCTTG CGTCTCCCGACTTG-3'; acetyl-CoA carboxylase (ACC) forward, 5'-CCTGGTTCCCTGCTTACCTG-3', reverse, 5'-GTGGGATTGGACGTGCTGTA-3'; and mechanistic target of rapamycin (mTOR) forward 5'-AGAGGTCGGCACTCC ACTAT-3' and reverse, 5'-TGGCCAGGCTTCTGAACAAA-3'.

Compound $C$ treatment. Primary cardiomyocytes were treated with compound C (cat. no. 62749-26-2; Sigma-Aldrich; Merck KGaA) at a concentration of $20 \mu \mathrm{M}$ or PBS for $24 \mathrm{~h}$ at $37^{\circ} \mathrm{C}$. Subsequently, rAd-NC or rAd-sh-TFEC was transfected into primary caridomycytes for $24 \mathrm{~h}$ and cells were collected for further study.

Western blotting. Proteins isolated from heart tissue or primary cardiomyocytes were extracted using a total protein extraction kit (Beijing Solarbio Science \& Technology Co., Ltd.) at room temperature. A BCA protein assay kit (Pierce; Thermo Fisher Scientific, Inc.) was used to determine the protein concentration.
Proteins $(40 \mu \mathrm{g})$ were separated by $12 \%$ SDS-PAGE and transferred onto PVDF membranes. The membranes were blocked with 5\% fat-free milk (Pierce; Thermo Fisher Scientific, Inc.) at room temperature for $2 \mathrm{~h}$. Membranes were incubated with primary antibodies against GAPDH (cat. no. ab8245; 1:3,000), phosphorylated (p)-AMPK (cat. no. ab133448; 1:1,000), AMPK (cat. no. ab131357; 1:1,000), p-ACC (cat. no. ab173583; 1:1,000), ACC (cat. no. ab222774; 1:1,000), p-mTOR (cat. no. ab109268; 1:1,000), mTOR (cat. no. ab134903; 1:1,000) (all from Abcam) and TFEC (cat. no. ab116167; 1:1,000; Abcam) overnight at $4^{\circ} \mathrm{C}$. The membranes were then washed with $0.1 \%$ TBST and incubated with horseradish peroxidase-conjugated goat anti-rabbit IgG (1:5,000; cat. no. ZB-2301; Beijing Zhongshan Golden Bridge Biotechnology Co., Ltd.) for $2 \mathrm{~h}$ at room temperature. Enhanced chemiluminescence reagent (GE Healthcare) was used to detect the signal on the membrane. Signals were detected using a Super ECL Plus kit (Nanjing KeyGen Biotech Co., Ltd.) and quantitative analysis was performed using UVP 7.0 software (UVP, LLC). Relative protein expressions were normalized to GAPDH. All experiments were repeated three times. ImageJ $1.43 \mathrm{~b}$ software (National Institutes of Health) was used for densitometry analysis.

Statistical analysis. Prism 7.0 (GraphPad Software, Inc.) was used for the quantification of all data. In all experiments, each measurement was performed at least in triplicate. The data were presented as the means \pm the standard error of the mean. Two-tailed unpaired Student's t-test was used for comparisons between two groups. Comparisons between more than three groups were made by one-way ANOVA followed by Bonferroni post hoc test for multiple comparisons. $\mathrm{P}<0.05$ was considered to indicate a statistically significant difference.

\section{Results}

TFEC is upregulated in the hypertrophic myocardium of mice subjected to TAC. Compared with the sham control, TAC increased the size of mouse hearts, but no significant changes in body weight were observed (Fig. 1A and B). Furthermore, the HW/BW ratio was significantly increased in TAC mice compared with those of the sham control (Fig. 1B). Furthermore, the cardiomyocyte size was significantly increased in the hypertrophic myocardia of mice subjected to TAC compared with sham control mice (Fig. 1C). An echocardiography study indicated that the left ventricular posterior wall diameter (LVPWd) and dp/dtmin were increased in mice subjected to TAC compared with the sham control mice (Fig. 1D, E and H); however, LVEF (\%) and dp/dtmax were decreased in mice subjected to TAC compared with sham control mice (Fig. 1F and G). The results from western blotting demonstrated that TFEC expression was significantly increased in the hypertrophic myocardia of mice subjected to TAC compared with the sham control mice (Fig. 1I).

TFEC is upregulated in the hypertrophic myocardia of mice treated with AngII. The weight of the hypertrophic myocardia was significantly increased in the AngII-treated group compared with the control group, but no significant changes were observed in the body weight of mice between the two 
A

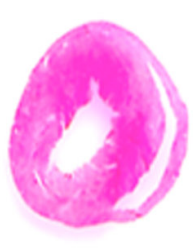

Sham
B

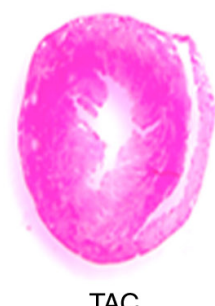

TAC

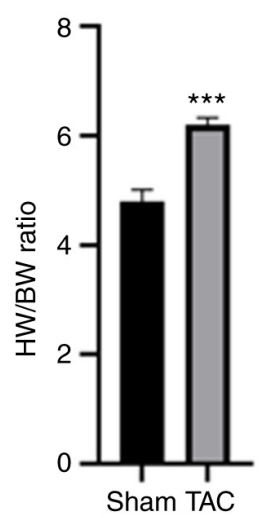

$E$

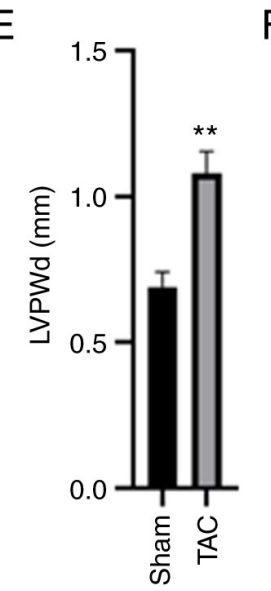

C

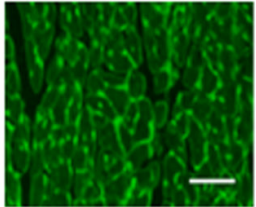

Sham

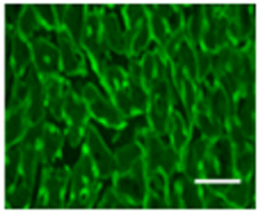

TAC $(20 x)$
$\mathrm{D}$

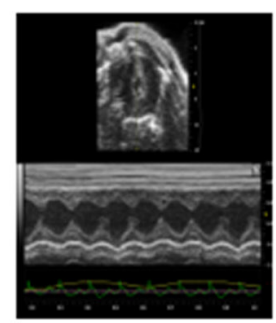

Sham

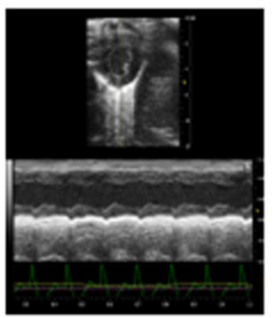

TAC
Sham

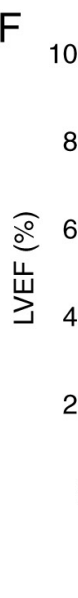

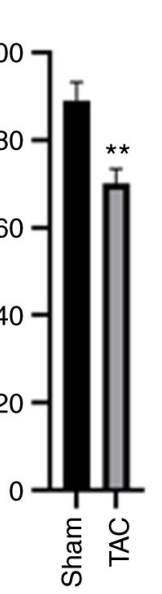

G

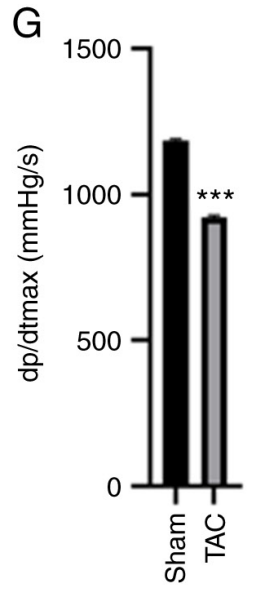

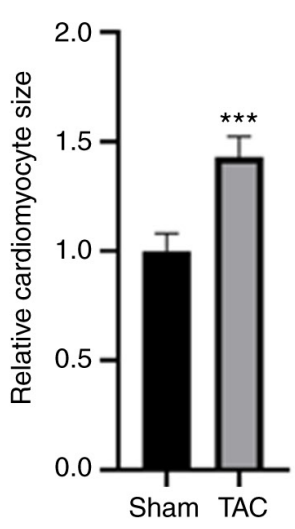

$\mathrm{H}$

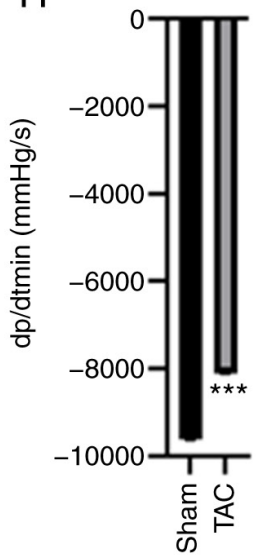

I
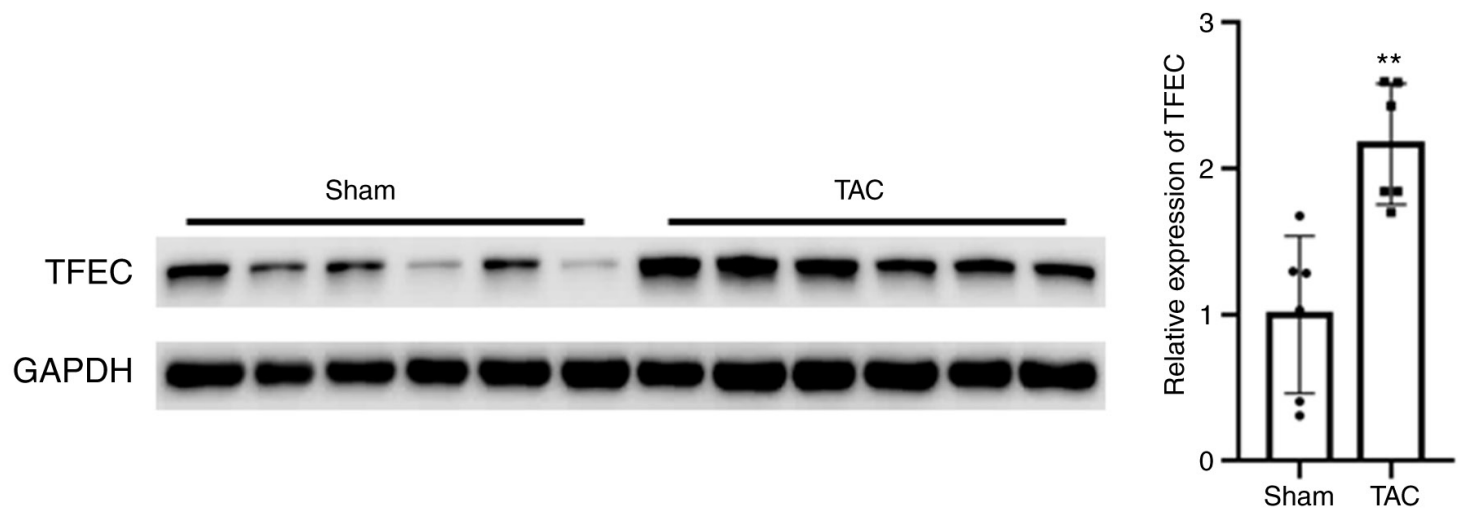

Figure 1. TFEC expression is increased in the hypertrophic myocardia of mice subjected to TAC. (A) Hematoxylin and eosin staining of heart tissues. (B) HW/BW was evaluated in the sham and TAC groups. (C) Wheat germ agglutinin staining showed that the cardiomyocyte size was significantly increased in the hypertrophic myocardia of mice subjected to TAC compared with control mice (magnification, x20). (D) Representative echocardiographic images. (E) LVPWd, (F) LVEF (\%), (G) dp/dtmax and (H) dp/dtmin were quantified in the hearts of TAC and sham control groups. (I) Western blotting showed that TFEC expression was increased in the hypertrophic myocardia of mice subjected to TAC compared with control mice. ${ }^{* * *} \mathrm{P}<0.01$ and ${ }^{* * * *} \mathrm{P}<0.001$. TAC, transverse aortic constriction; TFEC, transcription factor EC; HW/BW, heart weight and body weight ratio; LVEF, left ventricular ejection fractions; LVPWd, left ventricular posterior wall diameter; dp/dtmax, maximum change in left ventricular pressure over time; dp/dtmin, minimum change in left ventricular pressure over time.

groups (Fig. 2A and B). As presented in Fig. 2B, the HW/BW ratio was increased in the AngII treatment and control groups. The relative cardiomyocyte size was increased compared with that of the control group (Fig. 2C). Echocardiographic assays indicated that cardiac function was impaired in the hearts of AngII-treated mice, as indicated by elevated LVPWd and dp/dtmin and decreased LVEF (\%) and dp/dtmax (Fig. 2D-H). The results from western blotting demonstrated that TFEC expression was significantly increased in the hearts of AngII-treated mice compared with those of the control mice (Fig. 2I).
TFEC knockdown improves cardiac function in AngII-induced mice. The expression of TFEC was silenced in AngII-treated mice. As presented in Fig. 3A, compared with rAd-NC, injection with rAd-sh-TFEC significantly decreased the expression of TFEC in heart tissues. Furthermore, the HW/BW ratio and relative cardiomyocyte size were significantly decreased in mice injected with rAd-sh-TFEC compared with mice injected with rAd-NC (Fig. 3B and C). The expression levels of ANP, $\mathrm{BNP}$ and $\beta$-MHC were significantly increased in the hearts of AngII-treated mice; however, compared with transfection with rAd-NC, transfection with rAd-sh-TFEC decreased 
A

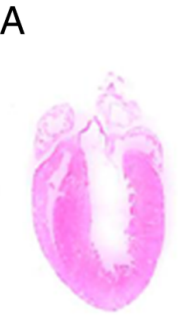

Con
$\mathrm{B}$

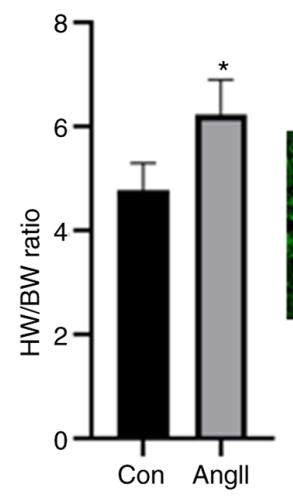

C

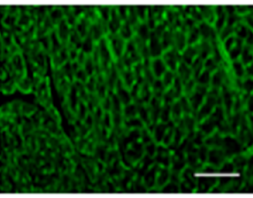

Con

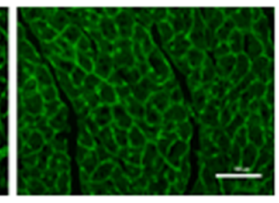

Angll

(20x)

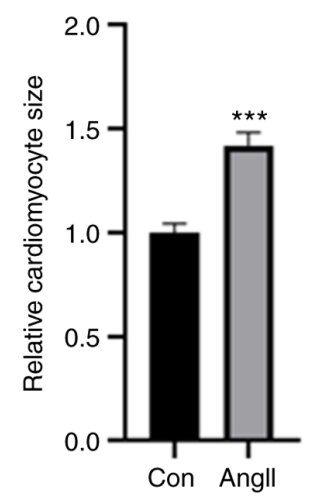

D

E

F

G

$\mathrm{H}$

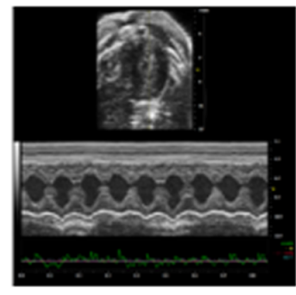

Con

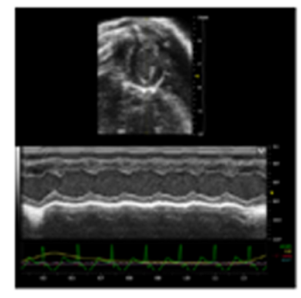

Angll
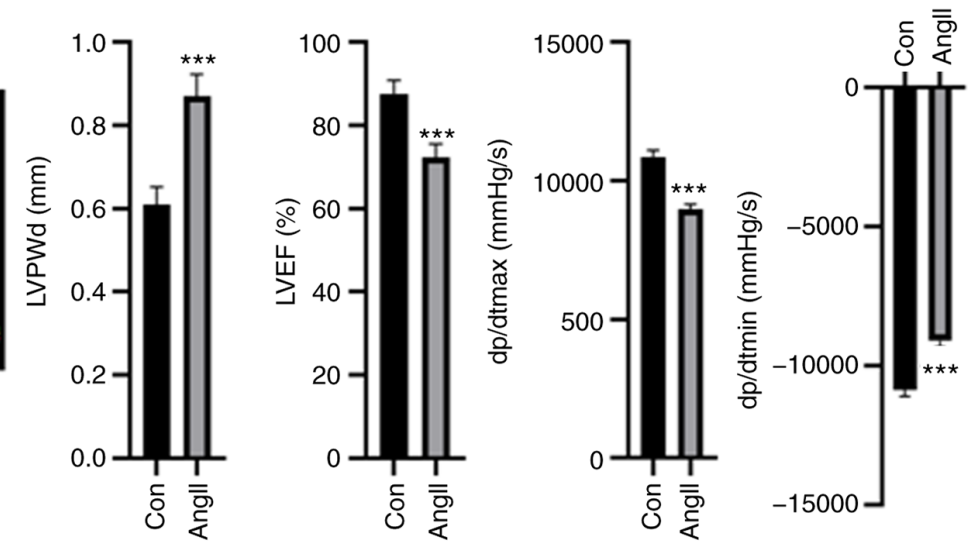

I
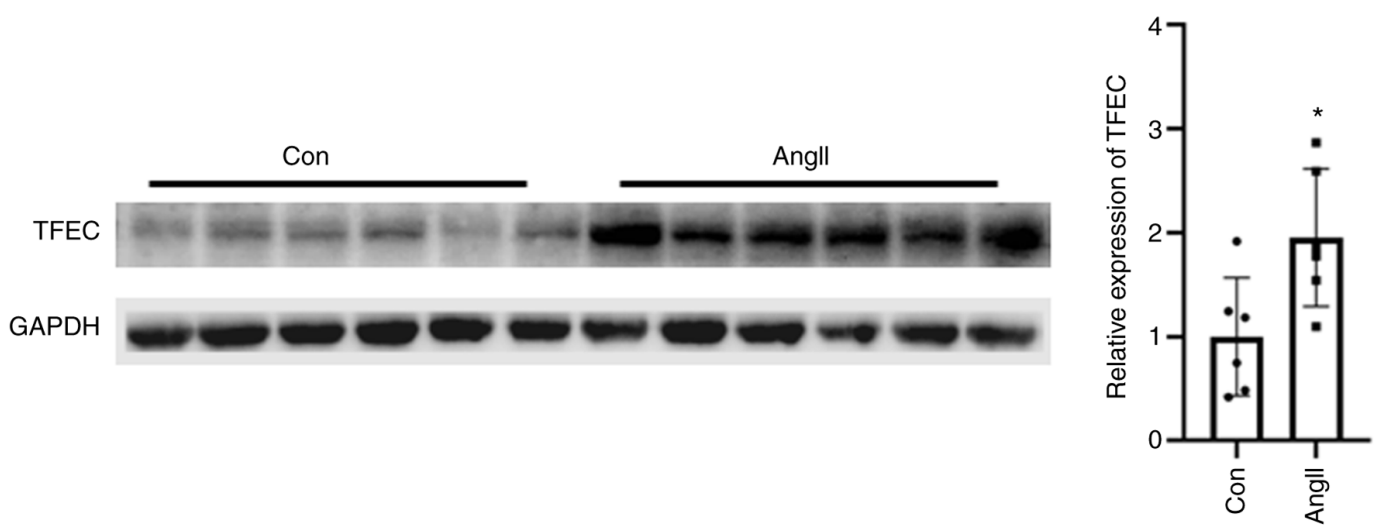

Figure 2. TFEC expression is elevated in the hearts of AngII-treated mice compared with control mice. (A) Hematoxylin and eosin staining of heart tissues. (B) HW/BW was evaluated in control and Ang-treated mice. (C) Wheat germ agglutinin staining was performed to evaluate the relative cardiomyocyte size (magnification, x20). (D) Representative echocardiographic images. (E) LVPWd, (F) LVEF (\%), (G) dp/dtmax and (H) dp/dtmin were quantified in the hearts of AngII-treated mice and sham control mice. (I) Western blotting assay demonstrated that TFEC expression was increased in the hearts of AngII-treated mice compared with those of control mice. ${ }^{*} \mathrm{P}<0.05$ and ${ }^{* * *} \mathrm{P}<0.001$. AngII, angiotensin II; TFEC, transcription factor EC; HW/BW, heart weight and body weight ratio; LVEF, left ventricular ejection fractions; LVPWd, left ventricular posterior wall diameter; dp/dtmax, maximum change in left ventricular pressure over time; dp/dtmin, minimum change in left ventricular pressure over time.

the expression levels of ANP, BNP and $\beta$-MHC (Fig. 3D). Echocardiographic assays indicated that transfection with rAd-sh-TFEC improved cardiac function in the hearts of AngII-treated mice compared with transfection with rAd-NC, as indicated by decreased LVPWd and dp/dtmin and increased LVEF (\%) and dp/dtmax (Fig. 3E-I).

Silencing TFEC abolishes AngII-induced cardiomyocyte hypertrophy. A cellular cardiomyocyte model was established. As demonstrated in Fig. 4A, treatment with AngII increased the relative cardiomyocyte size. The expression levels of ANP, BNP and $\beta$-MHC were significantly increased in primary cardiomyocytes treated with AngII compared with control cardiomyocytes (Fig. 4B). In primary cardiomyocytes, treatment with AngII significantly increased the expression of TFEC compared with the control (Fig. 4C). Furthermore, transfection with $\mathrm{rAd}$-sh-TFEC significantly reduced the expression of TFEC, even in primary cardiomyocytes treated with AngII (Fig. 4D). In addition, the AngII-induced increase in cardiomyocyte size could be reversed following TFEC knockdown in primary cardiomyocytes (Fig. 4E). The elevated expression levels of ANP, BNP and $\beta$-MHC induced 
A
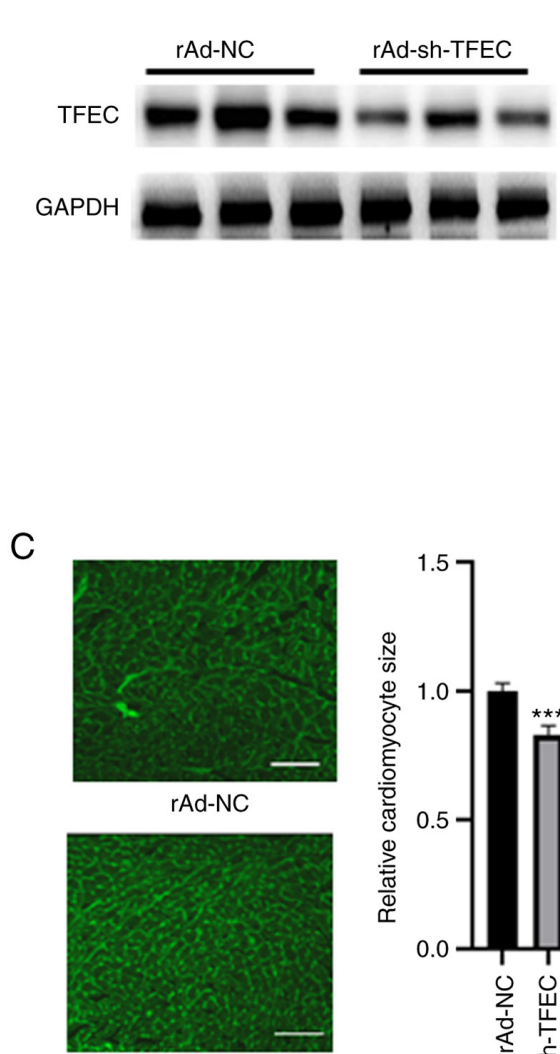

rAd-sh-TFEC (20x)

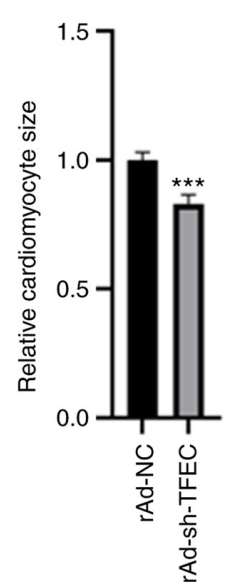

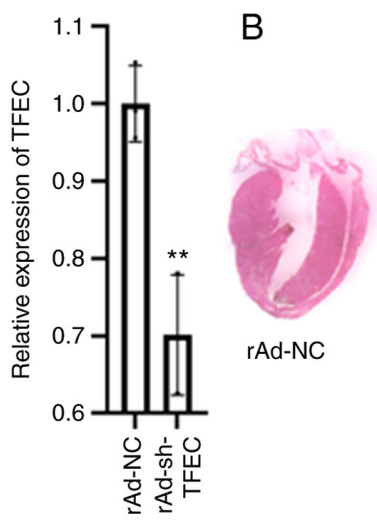
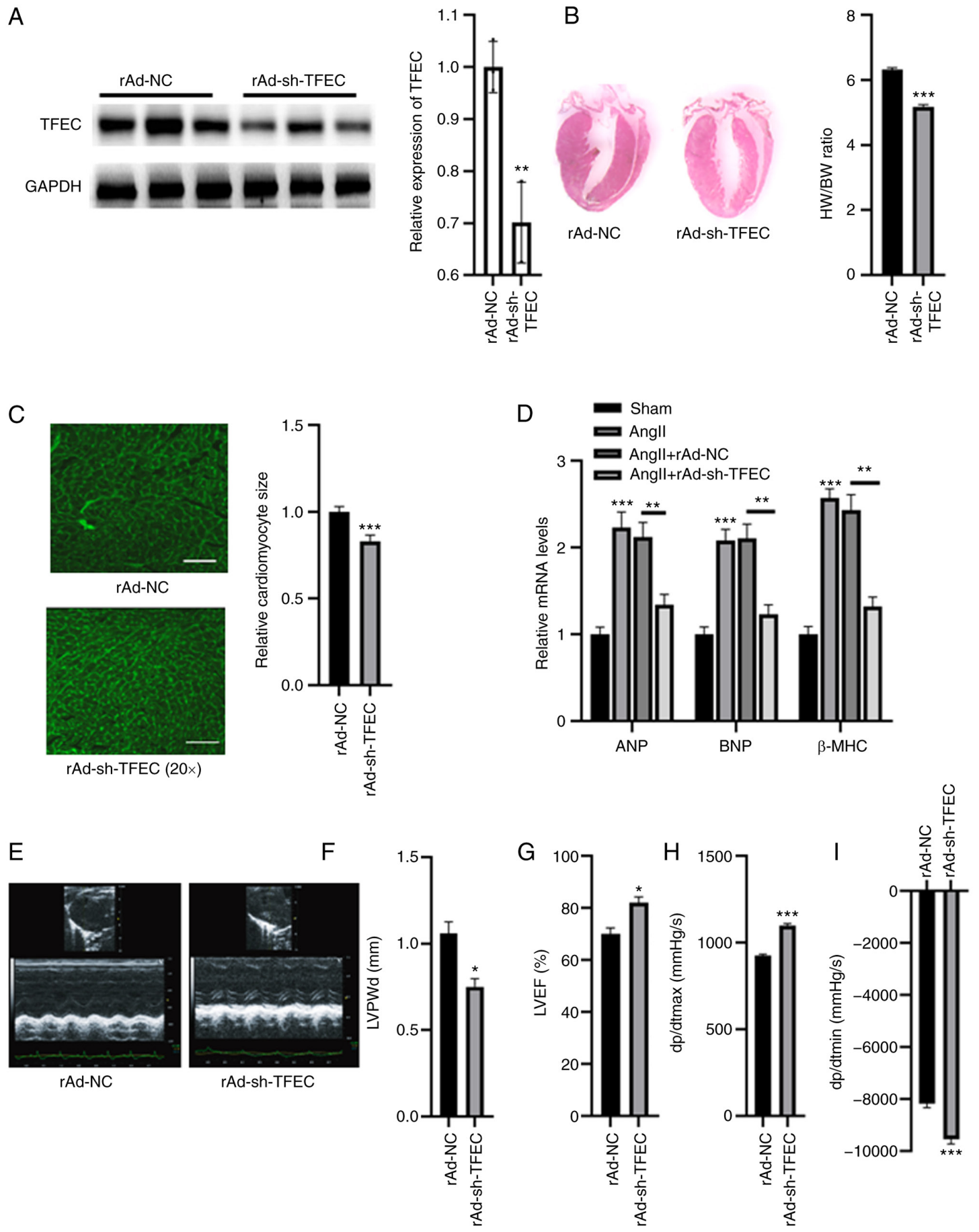

Figure 3. TFEC knockdown improves cardiac function in AngII-treated mice compared with NC mice. (A) Western blotting analysis showed that injection with rAd-sh-TFEC significantly decreased the expression of TFEC in heart tissues compared with injection with rAd-NC. (B) Knockdown of TFEC reduced the HW/BW compared with NC group. (C) Relative cardiomyocyte size was decreased in mice injected with rAd-sh-TFEC compared with those injected with rAd-NC (magnification, x20). (D) Transfection with rAd-sh-TFEC reduced the relative mRNA levels of ANP, BNP and $\beta$-MHC compared with transfection with rAd-NC. (E) Representative echocardiographic images. (F) LVPWd, (G) LVEF (\%), (H) dp/dtmax and (I) dp/dtmin were quantified in the hearts of rAd-sh-TFEC and rAd-NC-treated mice. ${ }^{*} \mathrm{P}<0.05,{ }^{* *} \mathrm{P}<0.01$ and ${ }^{* * * *} \mathrm{P}<0.001$. TFEC, transcription factor EC; sh, short hairpin; NC, negative control; HW/BW, heart weight and body weight ratio; ANP, atrial natriuretic peptide; BNP, brain natriuretic peptide; $\beta$-MHC, $\beta$-myosin heavy chain; LVEF, left ventricular ejection fractions; LVPWd, left ventricular posterior wall diameter; dp/dtmax, maximum change in left ventricular pressure over time; dp/dtmin, minimum change in left ventricular pressure over time.

by AngII could be partially abolished after TFEC knockdown (Fig. 4F).

TFEC inactivates AMPK/mTOR signaling. AMPK/mTOR signaling is suggested to play a key role in the AngII-induced cardiac hypertrophy model (18). Hence, we evaluated the effect of TFEC on AMPK/mTOR signaling. The results from western blotting demonstrated that TFEC expression was significantly increased in primary cardiomyocytes transfected with Ad-TFEC compared with that transfected with Ad-NC (Fig. 5A), indicating that transfection with rAd-TFEC overexpression vector was successful. The results from RT-qPCR 
A
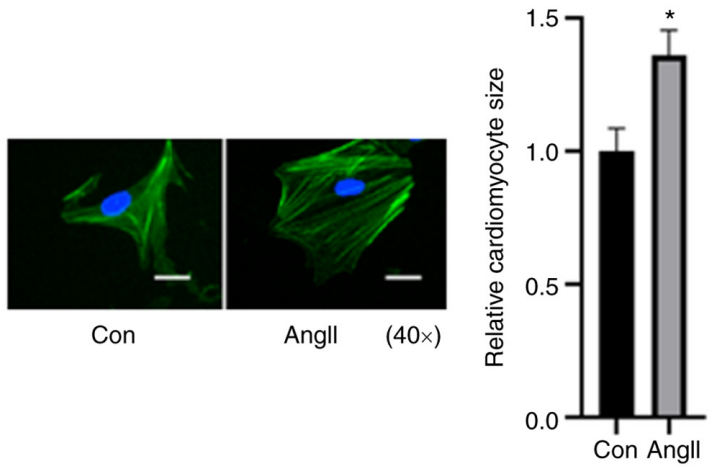

B

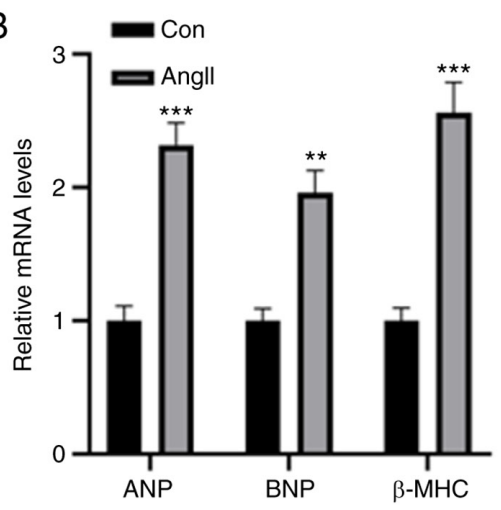

C

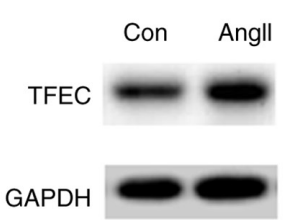

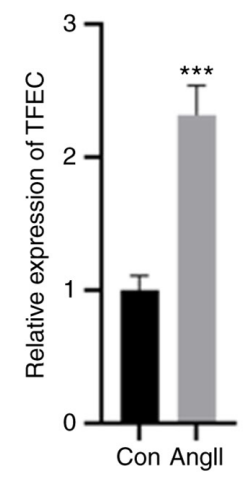

D
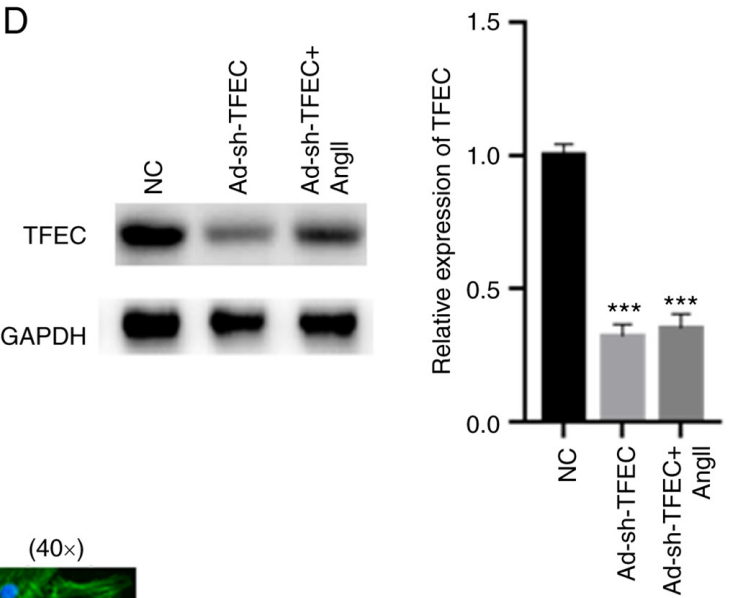

$\mathrm{E}$
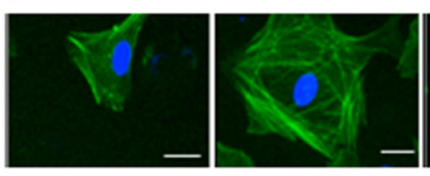

Angll

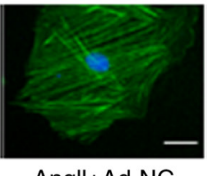

$(40 \times)$

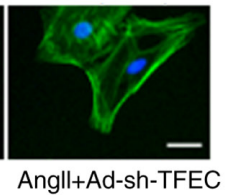

$\mathrm{F}$
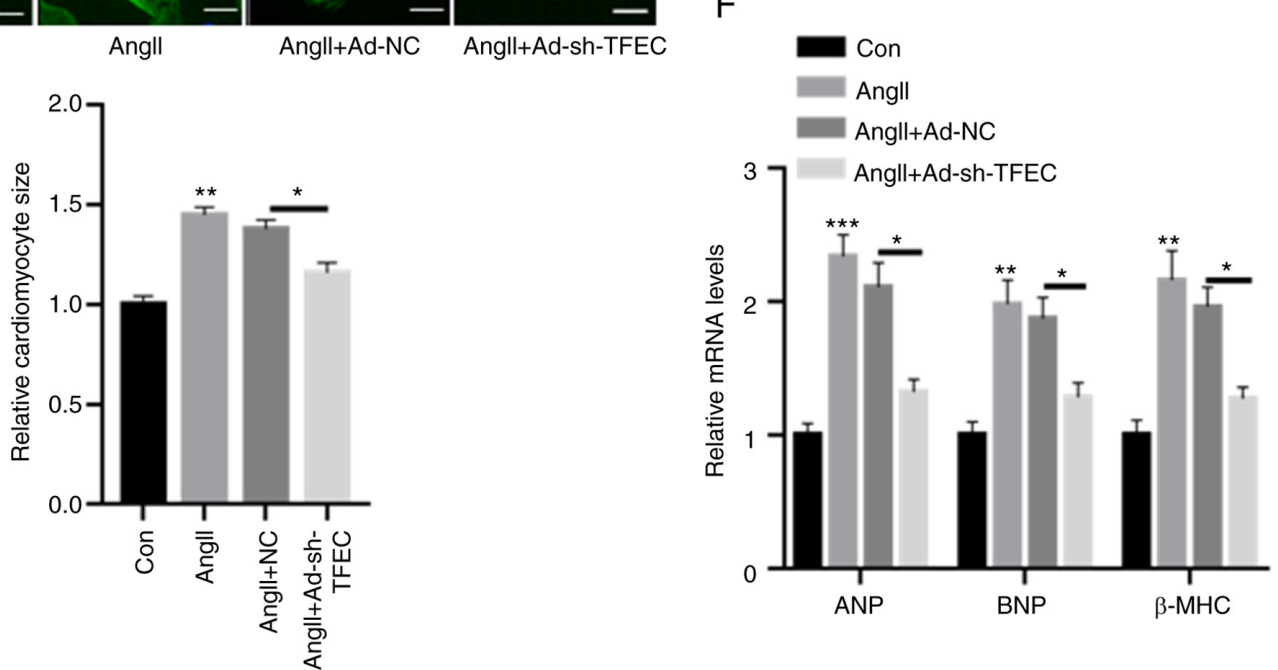

Figure 4. Silencing TFEC abolishes AngII-induced cardiomyocyte hypertrophy. (A) Compared with the control, treatment with AngII increased the relative cardiomyocyte size (magnification, x40). (B) mRNA levels of ANP, BNP and $\beta$-MHC were significantly increased in primary cardiomyocytes treated with AngII compared with the control. (C) In primary cardiomyocytes, treatment with AngII significantly increased the expression of TFEC compared with treatment with the control. (D) Transfection with rAd-sh-TFEC significantly decreased the expression of TFEC even in primary cardiomyocytes treated with AngII. (E) AngII-induced increase in cardiomyocyte size could be reversed by TFEC knockdown in primary cardiomyocytes (magnification, $\mathrm{x} 40$ ). (F) Elevated mRNA levels of ANP, BNP and $\beta$-MHC induced by AngII could be partially abolished following TFEC knockdown in primary cardiomyocytes. * $<<0.05$, ${ }^{* *} \mathrm{P}<0.01$ and ${ }^{* * *} \mathrm{P}<0.001$. AngII, angiotensin II; Con, control; NC, negative control; ANP, atrial natriuretic peptide; BNP, brain natriuretic peptide; $\beta$-MHC, $\beta$-myosin heavy chain; TFEC, transcription factor EC; sh, short hairpin.

demonstrated that transfection with rAd-TFEC did not change the expression levels of AMPK, ACC and mTOR, compared with transfection with rAd-NC (Fig. 5B). The results from western blotting showed that TFEC overexpression decreased the expression of p-AMPK and p-ACC but increased the expression of p-mTOR in primary cardiomyocytes (Fig. 5C). Conversely, silencing TFEC increased the expression of p-AMPK and p-ACC but decreased the level of p-mTOR (Fig. 5D). Furthermore, treatment with Compound C, which is an AMPK inhibitor, significantly inhibited the activation of 
A

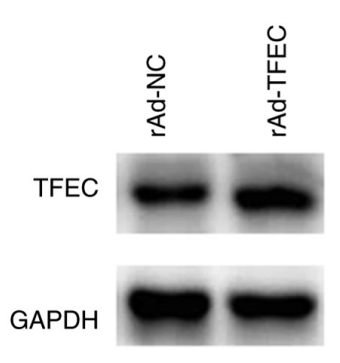

B

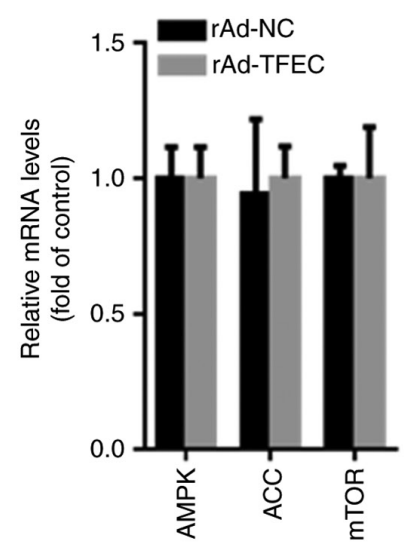

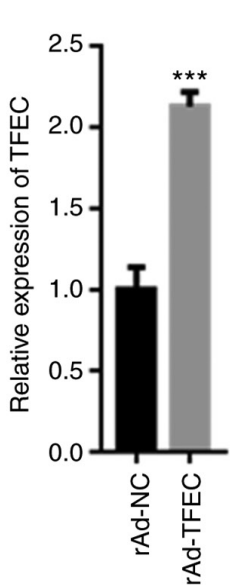

C

p-AMPK

AMPK

p-ACC

ACC
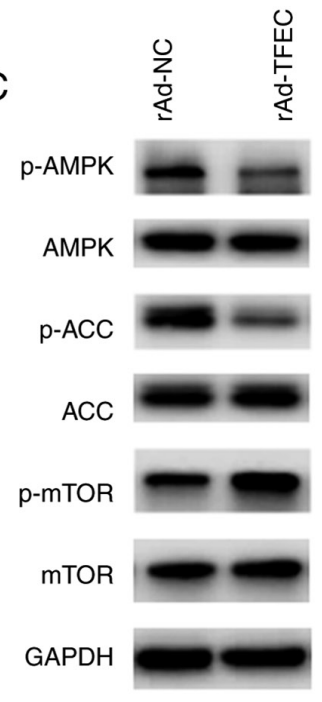

(1)

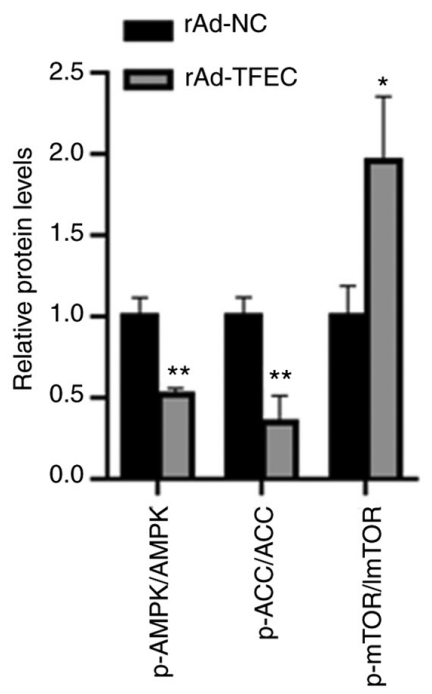

D

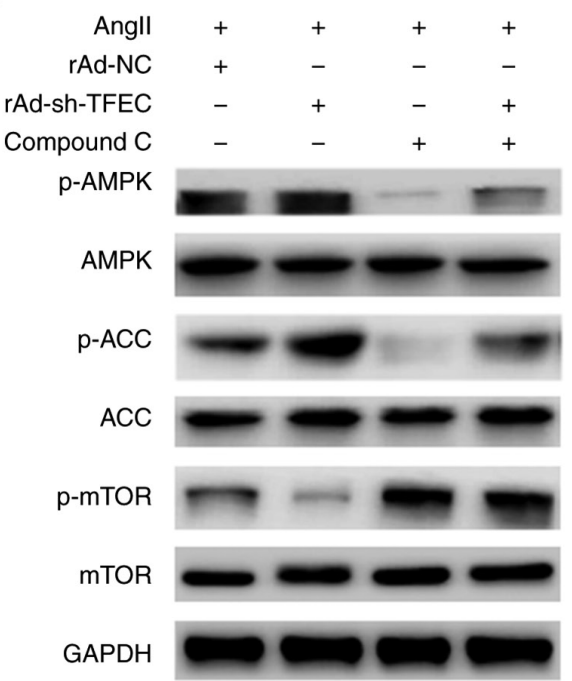

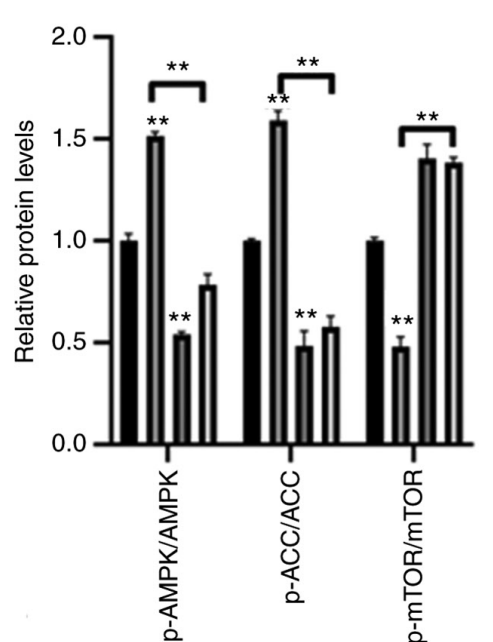

Figure 5. TFEC activates AMPK/mTOR signaling in primary cardiomyocytes. (A) Western blotting showed that TFEC expression was significantly increased in primary cardiomyocytes transfected with Ad-TFEC compared with those transfected with Ad-NC. (B) Reverse transcription quantitative PCR showed that transfection with rAd-TFEC did not change the mRNA levels of AMPK, ACC and mTOR, compared with transfection with rAd-NC. (C) Western blotting showed that TFEC overexpression decreased the expression of p-AMPK and p-ACC but increased the level of p-mTOR. (D) Compound C significantly suppressed the activation of AMPK/ACC but increased the activation of mTOR, even in primary cardiomyocytes transfected with rAd-sh-TFEC. "P<0.05, ${ }^{* *} \mathrm{P}<0.01$ and ${ }^{* * *} \mathrm{P}<0.001$. AMPK, AMP-activated protein kinase; mTOR, mechanistic target of rapamycin; ACC, acetyl-CoA carboxylase; NC, negative control; p, phosphorylated; sh, short hairpin; TFEC, transcription factor EC.

AMPK/ACC but increased the activation of mTOR, even in primary cardiomyocytes transfected with rAd-TFEC (Fig. 5D).

\section{Discussion}

Cardiac hypertrophy and fibrosis can be induced by many factors, including mechanical pressure overload and AngII (19). The present study explored therefore the expression of TFEC in a TAC-induced mouse model and a AngII-induced cardiac hypertrophy model. Elevated expression of TFEC was found in the hearts of mice with pressure overload- and AngII-induced hypertrophy. These observations suggested a potential detrimental role of TFEC in the pathology of cardiac hypertrophy.

AngII is the most important constituent of the renin-angiotensin aldosterone system, which serves a key role in regulating cardiomyocyte growth, cardiac hypertrophy and extracellular matrix accumulation (20-22). Thus, TFEC expression was silenced in the mouse model of AngII-induced cardiac hypertrophy. The results demonstrated that cardiac TFEC expression was decreased in rAd-sh-TFEC-transfected mice pretreated with AngII. In the process of cardiac remodeling, enlargement of the heart is a major and clear change that is tightly associated with hypertrophy and fibrosis (23). In the mouse model of AngII-induced cardiac remodeling, TFEC knockdown ameliorated cardiac hypertrophy by reducing cardiomyocyte enlargement and enhancing the HW/BW ratio. In addition, mice transfection with rAd-sh-TFEC protected the hearts from the pathological hypertrophy induced by AngII, since TFEC knockdown partially abolished the effects of AngII on cardiac hypertrophy.

AMPK signaling is suggested to be an important regulator of different physiological and pathological cellular events that 
occur in cardiovascular diseases (24-26). AMPK is a major checkpoint of cellular metabolism that enhances autophagy by inhibiting mTOR signaling $(18,27)$. AMPK/mTOR-mediated autophagy protects diabetic rats from myocardial damage (24). The present study demonstrated that TFEC overexpression inhibited AMPK/mTOR signaling in AngII-treated primary cardiomyocytes. Conversely, silencing TFEC induced an increase in $\mathrm{p}$-AMPK and $\mathrm{p}$-ACC expression and a decrease in $\mathrm{p}$-mTOR expression. However, following cotreatment with Compound $\mathrm{C}$, the effect of sh-TFEC on AMPK/mTOR signaling was abolished. It is possible that TFEC may induce myocardial injury and dysfunction at least in part via inhibition of AMPK/mTOR signaling. Previous studies have also reported that AMPK activation protects against the pathological progression of cardiac hypertrophy, ventricular remodeling and heart failure $(28,29)$. In the present study, we therefore hypothesized that the TFEC-mediated inactivation of AMPK/mTOR signaling may contribute to cardiac hypertrophy. However, the precise mechanisms by which TFEC-inactivated AMPK signaling could affect cardiac hypertrophy are not yet fully understood and require further investigation.

This study presented some limitations. It would be interesting to evaluate the serum concentration of cholesterol, triglyceride, HDL and LDL in mice, since these markers are known to be associated with heart function. In a future study, these biochemical parameters will be determined in the serum of pressure-overload cardiac hypertrophy and AngII infusion-induced cardiac hypertrophy animal models.

In conclusion, the findings from the present study demonstrated that TFEC was overexpressed in the hearts of mice with cardiac hypertrophy, and that silencing TFEC could improve AngII-induced cardiac hypertrophy and dysfunction by activating AMPK/mTOR signaling. These results suggested that TFEC may serve important roles in cardiac hypertrophy and dysfunction, providing potential therapeutic targets for heart failure.

\section{Acknowledgements}

Not applicable.

\section{Funding}

The present study was supported by a grant from the Weapon Industry 521 Hospital (grant no. WI-20180952).

\section{Availability of data and materials}

The datasets used and/or analyzed during the current study are available from the corresponding author on reasonable request.

\section{Authors' contributions}

$\mathrm{TZ}$ and $\mathrm{ZW}$ performed the experiments, analyzed the data and wrote the paper. YC and CN performed some of the RT-qPCR experiments. $\mathrm{XZ}$ designed the experiments, analyzed the data and gave final approval of the version to be published. All authors read and approved the final manuscript. TZ and XZ confirm the authenticity of all the raw data.

\section{Ethics approval and consent to participate}

The present study was approved by the Research Ethics Committee of Weapon Industry 521 Hospital (approval no. WI-J2018923A).

\section{Patient consent for publication}

Not applicable.

\section{Competing interests}

The authors declare that they have no competing interests.

\section{References}

1. Nakamura M and Sadoshima J: Mechanisms of physiological and pathological cardiac hypertrophy. Nat Rev Cardiol 15: 387-407, 2018.

2. Gallo S, Vitacolonna A, Bonzano A, Comoglio P and Crepaldi T: ERK: A key player in the pathophysiology of cardiac hypertrophy. Int J Mol Sci 20: 2164, 2019.

3. Meng R, Pei Z, Zhang A, Zhou Y, Cai X, Chen B, Liu G, Mai W, Wei $\mathbf{J}$ and Dong Y: AMPK activation enhances PPAR $\alpha$ activity to inhibit cardiac hypertrophy via ERK1/2 MAPK signaling pathway. Arch Biochem Biophys 511: 1-7, 2011.

4. Petratou K, Spencer SA, Kelsh RN and Lister JA: The MITF paralog tfec is required in neural crest development for fate specification of the iridophore lineage from a multipotent pigment cell progenitor. PLoS One 16: e0244794, 2021.

5. Ballesteros-Alvarez J, Dilshat R, Fock V, Möller K, Karl L, Larue L, Ögmundsdóttir MH and Steingrímsson E: MITF and TFEB cross-regulation in melanoma cells. PLoS One 15: e0238546, 2020.

6. Mahony CB, Fish RJ, Pasche C and Bertrand JY: Tfec controls the hematopoietic stem cell vascular niche during zebrafish embryogenesis. Blood 128: 1336-1345, 2016.

7. Rehli M, Sulzbacher S, Pape S, Ravasi T, Wells CA, Heinz S, Söllner L, El Chartouni C, Krause SW, Steingrimsson E, et al: Transcription factor Tfec contributes to the IL-4-inducible expression of a small group of genes in mouse macrophages including the granulocyte colony-stimulating factor receptor. J Immunol 174: 7111-7122, 2005.

8. Zhao GQ, Zhao Q, Zhou X, Mattei MG and de Crombrugghe B: TFEC, a basic helix-loop-helix protein, forms heterodimers with TFE3 and inhibits TFE3-dependent transcription activation. Mol Cell Biol 13: 4505-4512, 1993.

9. Sun S, Kee HJ, Jin L, Ryu Y, Choi SY, Kim GR and Jeong MH: Gentisic acid attenuates pressure overload-induced cardiac hypertrophy and fibrosis in mice through inhibition of the ERK1/2 pathway. J Cell Mol Med 22: 5964-5977, 2018.

10. Dutton JW III, Artwohl JE, Huang $X$ and Fortman JD: Assessment of pain associated with the injection of sodium pentobarbital in laboratory mice (Mus musculus). J Am Assoc Lab Anim Sci 58: 373-379, 2019.

11. Takayanagi T, Kawai T, Forrester SJ, Obama T, Tsuji T, Fukuda Y, Elliott KJ, Tilley DG, Davisson RL, Park JY and Eguchi S: Role of epidermal growth factor receptor and endoplasmic reticulum stress in vascular remodeling induced by angiotensin II. Hypertension 65: 1349-1355, 2015.

12. Kopechek JA, McTiernan CF, Chen X, Zhu J, Mburu M, Feroze R, Whitehurst DA, Lavery L, Cyriac J and Villanueva FS: Ultrasound and Microbubble-targeted delivery of a microRNA inhibitor to the heart suppresses cardiac hypertrophy and preserves cardiac function. Theranostics 9: 7088-7098, 2019.

13. Tachibana S, Chen C, Zhang OR, Schurr SV, Hill C, Li R, Manso AM, Zhang J, Andreyev A, Murphy AN, et al: Analyzing oxygen consumption rate in primary cultured mouse neonatal cardiomyocytes using an extracellular flux analyzer. J Vis Exp: Feb 13, 2019 (Epub ahead of print). doi: 10.3791/59052.

14. Baird RC, Li S, Wang H, Naga Prasad SV, Majdalany D, Perni U and Wu Q: Pregnancy-associated cardiac hypertrophy in Corin-deficient mice: Observations in a transgenic model of preeclampsia. Can J Cardiol 35: 68-76, 2019. 
15. Sun J, Hao W, Fillmore N, Ma H, Springer D, Yu ZX, Sadowska A, Garcia A, Chen R, Muniz-Medina V, et al: Human Relaxin-2 fusion protein treatment prevents and reverses isoproterenol-induced hypertrophy and fibrosis in mouse heart. J Am Heart Assoc 8: e013465, 2019.

16. Sundaresan NR, Gupta M, Kim G, Rajamohan SB, Isbatan A and Gupta MP: Sirt3 blocks the cardiac hypertrophic response by augmenting Foxo3a-dependent antioxidant defense mechanisms in mice. J Clin Invest 119: 2758-2771, 2009.

17. Livak KJ and Schmittgen TD: Analysis of relative gene expression data using real-time quantitative PCR and the 2(-Delta Delta C(T)) method. Methods 25: 402-408, 2001.

18. Yan J, Yan JY, Wang YX, Ling YN, Song XD, Wang SY, Liu HQ, Liu QC, Zhang Y, Yang PZ, et al: Spermidine-enhanced autophagic flux improves cardiac dysfunction following myocardial infarction by targeting the AMPK/mTOR signalling pathway. $\mathrm{Br}$ J Pharmacol 176: 3126-3142, 2019.

19. Zhai CG, Xu YY, Tie YY, Zhang Y, Chen WQ, Ji XP, Mao Y, Qiao L, Cheng J, Xu QB and Zhang C: DKK3 overexpression attenuates cardiac hypertrophy and fibrosis in an angiotensin-perfused animal model by regulating the ADAM17/ACE2 and GSK-3 $\beta / \beta$-catenin pathways. J Mol Cell Cardiol 114: 243-252, 2018

20. Hu S, Cheng M, Guo X, Wang S, Liu B, Jiang H, Huang C and Wu G: Down-regulation of miR-200c attenuates AngII-induced cardiac hypertrophy via targeting the MLCK-mediated pathway. J Cell Mol Med 23: 2505-2516, 2019.

21. Xiao YF, Zeng ZX, Guan XH, Wang LF, Wang CJ, Shi H, Shou W, Deng KY and Xin HB: FKBP12.6 protects heart from AngII-induced hypertrophy through inhibiting $\mathrm{Ca}^{2+} / \mathrm{calmod}-$ ulin-mediated signalling pathways in vivo and in vitro. J Cell Mol Med 22: 3638-3651, 2018.

22. Dai W, Chen H, Jiang J, Kong W and Wang Y: Silencing MR-1 attenuates inflammatory damage in mice heart induced by AngII. Biochem Biophys Res Commun 391: 1573-1578, 2010.
23. Liang J, Huang B, Yuan G, Chen Y, Liang F, Zeng H, Zheng S, Cao L, Geng D and Zhou S: Stretch-activated channel Piezol is up-regulated in failure heart and cardiomyocyte stimulated by AngII. Am J Transl Res 9: 2945-2955, 2017.

24. Ren PH, Zhang ZM, Wang P, Zhu HP and Li ZQ: Yangxinkang tablet protects against cardiac dysfunction and remodelling after myocardial infarction in rats through inhibition of AMPK/mTOR-mediated autophagy. Pharm Biol 58: 321-327, 2020.

25. Wang Y, Zhu S, Liu H, Wei W, Tu Y, Chen C, Song J, Li J, Sun S, Wang $\mathrm{C}$ and $\mathrm{Xu} \mathrm{Z}$ : Thyroxine alleviates energy failure, prevents myocardial cell apoptosis, and protects against doxorubicin-induced cardiac injury and cardiac dysfunction via the LKB1/AMPK/mTOR axis in mice. Dis Markers 2019: 7420196, 2019.

26. Zhao J, Chen D and Feng C: Troxerutin attenuates isoproterenol-induced cardiac hypertrophy via the LKB1/AMPK/mTOR pathway. Panminerva Med 63: 233-234, 2019.

27. Zhang J, Zhao P, Quan N, Wang L, Chen X, Cates C, Rousselle T and $\mathrm{Li} \mathrm{J}$ : The endotoxemia cardiac dysfunction is attenuated by AMPK/mTOR signaling pathway regulating autophagy. Biochem Biophys Res Commun 492: 520-527, 2017.

28. Majd S, Power JHT, Chataway TK and Grantham HJM: A comparison of LKB1/AMPK/mTOR metabolic axis response to global ischaemia in brain, heart, liver and kidney in a rat model of cardiac arrest. BMC Cell Biol 19: 7, 2018.

29. Qi H, Ren J, Ba L, Song C, Zhang Q, Cao Y, Shi P, Fu B, Liu Y and Sun H: MSTN attenuates cardiac hypertrophy through inhibition of excessive cardiac autophagy by blocking AMPK/mTOR and miR-128/PPAR $\gamma / \mathrm{NF}-\kappa \mathrm{B}$. Mol Ther Nucleic Acids 19: 507-522, 2020.

This work is licensed under a Creative Commons Attribution-NonCommercial-NoDerivatives 4.0 International (CC BY-NC-ND 4.0) License. 